\title{
LES HUIT PREMIERS TRAVAUX DE PIERRE LIARDET
}

\author{
MichEL WALDSCHMIDT
}

À la mémoire de Pierre Liardet

\begin{abstract}
Ce texte est une présentation résumée des huit premiers travaux de Pierre Liardet. Il reprend l'exposé donné à l'Université de Savoie Mont Blanc (Le Bourget-du-Lac) lors du colloque Théorie des Nombres, Systèmes de Numération, Théorie Ergodique les 28 et 29 septembre 2015, un colloque inspiré par les mathématiques de Pierre Liardet.

Le premier texte publié par Pierre Liardet l'a été en 1969 dans les Comptes Rendus de l'Académie des Sciences de Paris, il est intitulé "Transformations rationnelles laissant stables certains ensembles de nombres algébriques", avec Madeleine Ventadoux comme coauteur. Ils étendent des résultats de Gérard Rauzy.

Dans la lignée de ces premiers travaux, il s'est attaqué à une conjecture de Władysław Narkiewicz sur les transformations polynomiales et rationnelles. En 1976, avec Ken K. Kubota, il a finalement réfuté cette conjecture.

Il a ensuite obtenu des résultats précurseurs sur une conjecture de Serge Lang, qui sont très souvent cités. Nous donnerons un bref survol des résultats qui ont suivi cette percée significative.
\end{abstract}

\section{Communiqué par Jean-Louis Verger-Gaugry}

2010 Mathematics Subject Classification: Primary 11-03, 12-03, 14-03; Secundary 11D41, 11R04, 11R06, 11R09, 11R18, 11R99, 12D10, 12D99, 12E05, 12E99, 12F05, 12F99, 14E05, $14 \mathrm{G} 05$.

Keywords: Transformations polynomiales, transformations rationnelles, stabilité d'ensembles algébriques, conjecture de Narkiewicz, nombres de Pisot-Vijayaraghavan, propriété de Northcott, topologies hilbertiennes, équations diophantiennes exponentielles, points rationnels sur un groupe algébrique, géométrie diophantienne, conjecture de Lang, conjecture de Mordell-Lang, intersections exceptionnelles.

C'est un plaisir de remercier Claude Levesque, Władysław Narkiewicz, Michel Laurent et Umberto Zannier pour leurs remarques mathématiques et typographiques. 


\section{Transformations rationnelles laissant stables certains ensembles de nombres algébriques}

Soit $S$ l'ensemble des nombres de Pisot-Vijayaraghavan, c'est-à-dire des entiers algébriques réels $>1$ dont les autres conjugués ont un module inférieur à 1 . Il résulte de la définition que pour $\theta \in S$ et $m \in \mathbf{N}, m \geq 1$, on a $\pm \theta^{m} \in S$. G. Rauzy [34, 35] a démontré la réciproque suivante. Soit $f \in K(X)$ une fraction rationnelle à une indéterminée non constante définie sur un corps $K$ contenant $\mathrm{C}$ et soit $E$ un sous-ensemble de $S$ tel que $S \backslash E$ soit borné. Supposons que pour tout $\theta \in E$, on ait $f(\theta) \in S$. Alors il existe $m \in \mathbf{N}, m \geq 1$, tel que $f(X)= \pm X^{m}$.

Pour généraliser la situation, introduisons un entier $n \geq 1$ et définissons $\Theta_{n}$ comme l'ensemble des entiers algébriques de degré $\geq n$ ayant $n$ conjugués de module $>1$ et tous les autres de module $<1$. Ainsi $\Theta_{1}$ est l'ensemble des éléments de $S$ et de leurs conjugués complexes. De nouveau, pour $\theta \in \Theta_{n}$ et pour $m \geq 1$, on a $\pm \theta^{m} \in \Theta_{n}$. P. Liardet et M. Ventadoux [1] généralisent le résultat de Rauzy en démontrant que, réciproquement, si $f \in K[X]$ est un polynôme non constant à coefficients dans une extension $K$ de $\mathbf{C}$, si $E$ est un sous-ensemble de $\Theta_{n}$ tel que $\Theta_{n} \backslash E$ soit borné et si $f(E) \subset \Theta_{n}$, alors il existe $m \geq 1$ tel que $f(X)= \pm X^{m}$.

\section{Stabilité rationnelle}

\subsection{La propriété $(P)$}

Soit $K$ un corps. Suivant W. Narkiewicz [31], on dit que $K$ a la propriété $(P)$ si tout polynôme $f \in K[X]$, pour lequel il existe un ensemble infini $E \subset K$ avec $f(E)=E$, est nécessairement linéaire. La propriété $(P)$ résulte de la propriété de Northcott, à savoir que les sous-ensembles de points de hauteur bornée sont finis. Mais la réciproque n'est pas vraie.

P. Liardet [2] démontre que si $K$ a la propriété $(P)$ de même que toutes ses extensions de degré $\leq n$, alors les corps de fonctions sur $K$ de degré $\leq n$ l'ont aussi: pour tout $r \geq 1$ et toute extension $L$ de $K\left(X_{1}, \ldots, X_{r}\right)$ de degré $\leq n$, le corps $L$ a la propriété $(P)$. Ceci étend un résultat de W. Narkiewicz [31] pour les corps de nombres.

P. Liardet introduit aussi la définition suivante: un corps $K$ a la propriété $\left(P^{\prime}\right)$ si tout polynôme $f \in K[X]$ pour lequel il existe un sous-ensemble infini $E$ de la clôture algébrique $\bar{K}$ de $K$ avec $\max _{\alpha \in E}[K(\alpha): K]<\infty$ et $E=f(E)$ est nécessairement linéaire. Il démontre que si $K$ a la propriété $\left(P^{\prime}\right)$, alors toute extension finie de $K$ aussi, et toute extension transcendante pure aussi. Il étend ces résultats non seulement aux fractions rationnelles mais aussi en plusieurs variables. 


\section{LES HUIT PREMIERS TRAVAUX DE PIERRE LIARDET}

Dans sa note [3], P. Liardet considère des applications rationnelles $f$ et $g$ sur un corps $K$ ayant la propriété de Northcott, avec $\operatorname{deg} f>\operatorname{deg} g$, et montre, sous certaines conditions sur les zéros de $f$, que si $g$ est injective sur un sous-ensemble $\mathcal{X}$ de $K$ et si $g(\mathcal{X}) \subset f(\mathcal{X})$, alors $\mathcal{X}$ est fini. Dans leur texte [25], Halter-Koch et Narkiewicz affaiblissent les hypothèses sur les zéros dans le cas des polynômes.

\subsection{La conjecture de Narkiewicz}

W. Narkiewicz [30] a conjecturé la caractérisation suivante des corps ayant la propriété $(P)$ : un corps $K$ a la propriété $(P)$ si et seulement s'il existe une extension transcendante pure $L$ du sous-corps premier $F$ de $K$ et un ensemble $\mathcal{A}$ d'éléments de $L$ de degrés bornés sur $K$ tel que $L=K(\mathcal{A})$. K. K. Kubota et P. Liardet 4 réfutent cette conjecture: ils construisent des corps ayant la propriété $\left(P^{\prime}\right)$ (donc à plus forte raison la propriété $(P)$ ) qui ne sont pas de cette forme.

Ils montrent aussi l'existence d'un ensemble infini $\mathcal{P}$ de nombres premiers tel que le corps $\mathbf{Q}(\{\sqrt{p} \mid p \in \mathcal{P}\})$ ait la propriété $\left(P^{\prime}\right)$. Ils posent la question de savoir si ce résultat est encore vrai quand on prend pour $\mathcal{P}$ l'ensemble de tous les nombres premiers. La réponse, positive, a été apportée par Dvornicich et Zannier en 2007 22. Dans cet article, Dvornicich et Zannier donnent un exemple d'extension finie $L / K$ dans laquelle $L$ n'a pas la propriété $(P)$ bien que $K$ l'ait. Ils ont écrit un article de synthèse sur ces questions en 2008 [23] dans lequel ils relient la propriété de Northcott et la propriété $(P)$ au comportement des points pré-périodiques d'applications polynomiales.

On déduit des travaux de Bombieri et Zannier [19] que non seulement le corps engendré par tous les nombres $\sqrt{p}, p$ décrivant l'ensemble des nombres premiers, a la propriété $(P)$, mais qu'il en est de même pour le corps engendré par toutes les racines $n$-ièmes des entiers rationnels, premiers ou non.

La note 4] de K. K. Kubota et P. Liardet est citée non seulement dans l'article de Dvornicich et Zannier [22, mais aussi dans ceux de L. Pottmeyer [33] et M. Widmer [39].

\subsection{Stabilité algébrique et topologies hilbertiennes}

Soient $\Omega$ un corps, $A$ et $B$ deux sous-ensembles de $\Omega$. On désigne par $\mathcal{P}(A, B)$ l'ensemble des polynômes $f \in \Omega[X, Y]$ vérifiant:

(1) Pour tout $a \in A$ en dehors d'un ensemble fini, il existe $b \in B$ tel que $f(a, b)=0$.

(2) Pour tout $b \in B$, le polynôme $f(X, b) \in \Omega[X]$ n'est pas le polynôme nul.

Ces ensembles ont été étudiés par de nombreux auteurs parmi lesquels P. Liardet lui-même [6] cite D. J. Lewis, W. Narkiewicz, S. Lang, G. Rauzy, 
A. Schinzel, C. L. Siegel. G. Rauzy [34, 35] a démontré que si $f \in \mathcal{P}(S, S)$ où $S$ est, comme ci-dessus, l'ensemble des nombres de Pisot-Vijayaraghavan, alors il existe $m>0$ tel que $f\left(X, X^{m}\right)=0$ dans $\Omega[X]$. Résolvant une conjecture de Rauzy, P. Liardet [5, 6] obtient la même conclusion pour $f \in \mathcal{P}(T, T)$ où $T$ est l'ensemble des nombres de Salem (à savoir les nombres réels $>1$ dont tous les conjugués ont un module $\leq 1$ et un au moins est de module 1 ). Dans sa thèse en 1975 10, P. Liardet obtient de nombreux résultats sur ces ensembles $\mathcal{P}(A, B)$. Sa méthode consiste à introduire des nouvelles topologies qu'il appelle topologies hilbertiennes, en raison de leur lien avec le théorème d'irréductibilité de Hilbert. Il étudie aussi l'analogue de ses résultats dans les corps de séries formelles de Laurent, avec les éléments P-V de P. Bateman et A. Duquette [14].

Ses résultats ont été appliqués récemment pour étudier des ensembles ayant la propriété de Northcott, notamment par S. Checcoli et M. Widmer [21, 39].

\section{Sur une conjecture de Serge Lang}

Un des principes heuristiques de base en géométrie diophantienne est que les points rationnels sur une variété algébrique sont gouvernés par la géométrie. De nombreux exemples justifient ce principe. L'existence de points entiers ou rationnels sur une courbe algébrique dépend de son genre (théorème de Siegel pour les points entiers sur un corps de nombres, conjecture de Mordell démontrée par Faltings pour les points rationnels). S. Lang [26, 27, 28] a été un précurseur dans la recherche d'énoncés généraux, ne tenant aucun compte des méthodes disponibles, mais cherchant, avec une remarquable intuition, à décrire la situation générale de façon "fonctorielle" et cohérente.

\subsection{Equations Diophantiennes exponentielles}

Une généralisation de la notion d'équation diophantienne est celle d'équation diophantienne exponentielle, dans laquelle certains exposants sont des inconnues. On l'interprète géométriquement en considérant des points entiers ou rationnels sur une courbe dans un groupe algébrique.

La conjecture de Mordell-Lang en caractéristique zéro s'énonce ainsi [37]:

Soit $X$ une sous-variété fermée géométriquement irréductible d'une variété semi-abélienne A définie sur un corps de caractéristique nulle. Soit $\Gamma$ un sous-groupe de rang fini de $A(K)$. Si $X$ n'est pas un translaté d'une sous-variété semi-abélienne de A, alors $X(K) \cap \Gamma$ n'est pas Zariski dense dans $X$. 


\subsection{La contribution de P. Liardet}

Prenons comme exemple le polynôme $f(X, Y)=X^{5} Y+3 X^{3} Y^{4}+2 X Y^{7}$. L'équation

$$
f(x, y)=0
$$

admet comme solutions rationnelles

$$
(x, y)=\left(5^{3 a},-5^{2 a}\right), \quad a \in \mathbf{Z} .
$$

Cela signifie que l'intersection de la courbe définie par cette équation avec le sousgroupe multiplicatif de $\left(\mathbf{Q}^{\times}\right)^{2}$ de $\mathbf{G}_{m}^{2}$ engendré par $\left(5^{3},-5^{2}\right)$ est infinie. La raison en est que le polynôme $f\left(t^{3},-t^{2}\right)$ est nul.

C'est un fait général, démontré par P. Liardet: au lieu de

$$
\left\{\left(5^{3 a},-5^{2 a}\right), \quad a \in \mathbf{Z}\right\} \subset\left(\mathbf{Q}^{\times}\right)^{2},
$$

il considère plus généralement le groupe de division d'un sous-groupe de type fini de $\left(\mathbf{C}^{\times}\right)^{2}$. Soit $\Gamma_{0}$ un sous-groupe de type fini de $\left(\mathbf{C}^{\times}\right)^{2}: \Gamma_{0}$ est engendré par un sous-ensemble fini $\left\{\gamma_{1}, \gamma_{2}, \ldots, \gamma_{r}\right\}$ de $\left(\mathbf{C}^{\times}\right)^{2}$ :

$$
\Gamma=\left\{\gamma_{1}^{a_{1}} \ldots \gamma_{r}^{a_{r}} \mid\left(a_{1}, \ldots, a_{r}\right) \in \mathbf{Z}^{r}\right\} \subset\left(\mathbf{C}^{\times}\right)^{2} .
$$

Soit $\Gamma$ le groupe de division de $\Gamma_{0}$ :

$$
\Gamma=\left\{\gamma \in\left(\mathbf{C}^{\times}\right)^{2} \mid \text { il existe } m \in \mathbf{Z}, m \neq 0 \text {, tel que } \gamma^{m} \in \Gamma\right\} .
$$

ThÉonème ([7, 8]). Si $f(X, Y) \in \mathbf{C}[X, Y]$ vérifie $f(\gamma)=0$ pour une infinité de $\gamma \in \Gamma$, alors il existe $(a, b) \in \Gamma^{2}$ et $(u, v) \in \mathbf{Z}^{2}$ tels que $f\left(a T^{u}, b T^{v}\right)=0$ dans $\mathbf{C}(T)$.

Les sous-groupes algébriques connexes de $\mathbf{G}_{m}^{2}$ sont $\{1\}, \mathbf{G}_{m}^{2}$, et les sousgroupes de la forme

$$
H_{u, v}=\left\{\left(t^{u}, t^{v}\right) \mid t \in K^{\times}\right\}
$$

avec $(u, v) \in \mathbf{Z}^{2}, \operatorname{pgcd}(u, v)=1$. Ce théorème de Liardet peut donc aussi s'énoncer:

Si une courbe algébrique $C$ dans $G=\mathbf{G}_{m}^{2}$ ne contient pas de translaté d'un sous-groupe algébrique de dimension positive, et si $\Gamma$ est un sousgroupe de rang fini de $G(K)$, alors $\Gamma \cap C$ est fini.

S. Lang a suggéré un tel énoncé dès 1962 [26] et a démontré le cas particulier de ce théorème dans le cas où $\Gamma_{0}=\{1\}$ (donc $\Gamma$ est le sous-groupe de torsion de $\mathbf{G}_{m}(K)^{2}$ ) et aussi dans le cas où $\Gamma_{0} \cap C$ est infini. 


\subsection{Groupes algébriques commutatifs}

On dispose maintenant d'un énoncé général valable pour les variétés semiabéliennes, qui sont les extensions d'une variété abélienne par un tore:

Si A est une variété semi-abélienne sur un corps $K$ de caractéristique nulle, si $V$ est une sous-variété algébrique de $A$ et si $\Gamma$ est un sousgroupe de rang fini de $A(K)$, alors $\Gamma \cap V$ est une réunion finie

$$
V \cap \Gamma=\bigcup_{t+H \subset V}(t+H) \cap \Gamma
$$

où $t+H$ est un translaté d'un sous-groupe algébrique $H$ de $A$ contenu dans $V$.

M. Laurent 29] a obtenu ce résultat en 1984 quand $A$ est un tore multiplicatif $\mathbf{G}_{m}^{n}$; il donne une description explicite des sous-groupes $H$ qui interviennent dans la conclusion (ils sont associés aux partitions du support des équations). Un cas particulier où on peut assurer que $H=\{1\}$ est le suivant:

Si une sous-variété $V$ du groupe algébrique $G=\mathbf{G}_{m}^{n}$ ne contient pas de translaté d'un sous-groupe algébrique de dimension positive et si $\Gamma$ est un sous-groupe de rang fini de $G(K)$, alors $\Gamma \cap V$ est fini.

L'analogue du théorème de Laurent pour les variétés abéliennes a été obtenu par G. Faltings en 1991 (suite à des travaux de M. Raynaud sur la conjecture de Manin-Mumford en 1983). L'énoncé sur les variétés semi-abéliennes a été obtenu par M. Hindry (1988), M. McQuillan (1995) et P. Vojta (1996).

\subsection{Conjectures de géométrie diophantienne}

Après celles de Lang, une multitude de conjectures ont été proposées. Les plus connues sont celles de Manin-Mumford, André-Oort, Zilber, Zhang, Pink, Bogomolov. De nombreux travaux leur ont été consacrées — deux exposés au Séminaire Bourbaki en 2011 par A. Chambert-Loir [20] et par T. Scanlon [36] font le point sur ces questions, mais la théorie a encore beaucoup évolué depuis [38.

Les travaux de Zilber qui ont conduit à sa conjecture sur les intersections exceptionnelles ou atypiques trouve son origine dans son étude de la conjecture de Schanuel dans le cadre des corps différentiels. Les intersections exceptionnelles apparaissent antérieurement dans l'article de E. Bombieri, D.W. Masser et U. Zannier [17, dont le point de départ est un exemple simple de question conduisant à étudier l'intersection d'une courbe avec des sous-groupes algébriques de groupes multiplicatifs: ils considèrent les unités $\tau$ telles que $\tau$ et $1-\tau$ soient multiplicativement dépendants.

La théorie des modèles (o-minimalité) joue un rôle important dans les progrès les plus récents. 


\section{LES HUIT PREMIERS TRAVAUX DE PIERRE LIARDET}

La contribution [7, 8] de P. Liardet à la conjecture de Lang est citée dans les articles de I. Aliev et C. Smyth [13, A. Bérczes, [15], A. Bérczes, K. Győry, J.-H. Evertse et C. Pontreau [16], E. Bombieri, D. W. Masser et U. Zannier [18, J. Pila [32], P. Tzermias [37, ainsi que dans l'exposé de A. Chambert-Loir [20], dans le livre de U. Zannier sur les intersections exceptionnelles [40, Chap. 1, p. 22] et dans celui de J.-H. Evertse et K. Győry sur les équations aux unités [24, p. 321]. Le travail de Liardet sur la conjecture de Lang se situe donc au tout début d'une histoire riche, actuellement en plein développement.

La liste ci-dessous commence par des références aux huit premiers travaux de Pierre Liardet, continue par des références à sa thèse de troisième cycle [9] et à sa thèse d'État [10]. Les textes [11] et [12] ont été écrits en la mémoire de Pierre.

\section{BIBLIOGRAPHIE}

[1] VENTADOUX, M.-LIARDET, P.: Transformations rationnelles laissant stables certains ensembles de nombres algébriques, C. R. Acad. Sci. Paris Sér. A-B 269 (1969), A181-A183.

[2] LIARDET, P.: Sur les transformations polynomiales et rationnelles, in Séminaire de Théorie des Nombres, 1971-1972 (Univ. Bordeaux I, Talence), Exp. No. 29, Lab. Théorie des Nombres, Centre Nat. Recherche Sci., Talence, 1972, p. 20.

[3] _ : Sur une conjecture de W. Narkiewicz: C. R. Acad. Sci. Paris Sér. A-B 274 (1972), A1836-A1838.

[4] KUBOTA,K. K.-LIARDET, P.: Réfutation d'une conjecture de W. Narkiewicz, C. R. Acad. Sci. Paris Sér. A-B 282 (1976), no. 22, A1261-A1264.

[5] LIARDET, P.: Résultats de stabilité algébrique, in Séminaire de Théorie des Nombres, 1975-1976, (Univ. Bordeaux I, Talence), Exp. No. 24, Lab. Théorie des Nombres, Centre Nat. Recherche Sci., Talence, 1976, p. 6.

[6] __ Stabilité algébrique et topologies hilbertiennes, in Séminaire Delange-PisotPoitou, 17e année (1975/76), Théorie des nombres: Fasc. 1, Exp. No. 8, Secrétariat Math., Paris, 1977, p. 9.

[7] _ Sur une conjecture de Serge Lang, C. R. Acad. Sci. Paris Sér. A 279 (1974), $435-437$.

[8] _ Sur une conjecture de Serge Lang, in Journées Arithmétiques de Bordeaux (Conf., Univ. Bordeaux, Bordeaux, 1974), Soc. Math. France, Paris, 1975, 187-210. Astérisque, Nos. 24-25.

[9] _ : Transformations Rationnelles et Ensembles Algébriques, Thèse 3e cycle, Université de Provence, Faculté des Sciences 1970.

[10] __ : Première thèse: Sur la Stabilité Rationnelle ou Algébrique d'ensembles de Nombres Algébriques, Deuxième thèse; Difféomorphismes du Tore : Théorie Classique et Théorie Générique, Thèse d'État: Sciences mathématiques, Université d'Aix-Marseille II, Faculté des Sciences 1975. 


\section{MICHEL WALDSCHMIDT}

[11] ALLOUCHE, J.-P.-DAUDÉ, H.: Pierre Liardet (19432014), Gazette SMF 142, octobre 2014, 111-113.

http://smf4.emath.fr/Publications/Gazette/2014/142/smf_gazette_142_111-113.pdf

[12] BARAT, G.-GRABNER, P.J.-HELLEKALEK, P.: Pierre Liardet (1943-2014) in memoriam; EMS Newsletter September 2015, issue 97, 52-58.

https://www.ems-ph.org/journals/newsletter/pdf/2015-09-97.pdf

[13] ALIEV, I.-SMYTH, C.: Solving algebraic equations in roots of unity, Forum Math. 24 (2012), no. 3, 641-665.

[14] BATEMAn, P. T.-DUQUETTE, A. L.: The analogue of the Pisot-Vijayaraghavan numbers in fields of formal power series, Illinois J. Math. 6 (1962), 594-606.

[15] BÉRCZES, A.: Effective results for division points on curves in $\mathbb{G}_{m}^{2}$, J. Théor. Nombres Bordeaux 27 (2015), no. 2, 405-437.

[16] BÉRCZES, A.-GYÖRY, K.- EVERTSE, J.-H.-PONTREAU, C.: Effective results for points on certain subvarieties of tori, Math. Proc. Cambridge Philos. Soc. 147 (2009), no. 1, 69-94.

[17] BOMBIERI, E.-MASSER, D.-ZANNIER, U.: Intersecting a curve with algebraic subgroups of multiplicative groups, Internat. Math. Res. Notices 20 (1999), 1119-1140.

[18] _ Intersecting curves and algebraic subgroups: conjectures and more results, Trans. Amer. Math. Soc. 358 (2006), no. 5, 2247-2257.

[19] BOMBIERI, E.-ZANNIER, U.: A note on heights in certain infinite extensions of $\mathbb{Q}$, Atti Accad. Naz. Lincei Cl. Sci. Fis. Mat. Natur. Rend. Lincei (9) Mat. Appl. 12 (2001), 5-14 (2002).

[20] CHAMBERT-LOIR, A.: Relations de dépendance et intersections exceptionnelles, Séminaire Bourbaki Vol. 2010/2011. Exposés 1027-1042. Astérisque (2012), no. 348, Exp. No. 1032, viii, 149-188.

[21] CHECCOLI, S.-WIDMER, M.: On the Northcott property and other properties related to polynomial mappings, Math. Proc. Cambridge Philos. Soc. 155 (2013), no. 1, 1-12.

[22] DVORNICICH, R.-ZANNIER, U.: : Cyclotomic Diophantine problems (Hilbert irreducibility and invariant sets for polynomial maps), Duke Math. J. 139 (2007), no. 3, $527-554$.

[23] __ : On the properties of Northcott and of Narkiewicz for fields of algebraic numbers, Funct. Approx. Comment. Math. 39 (2008), no. 1, 163-173.

[24] EVERTSE, J.-H.- K. GYÖRY, K.: Unit Equation in Diophantine Number Theory, Cambridge Studies in Advanced Mathematics 146, Cambridge Univ. Press, 2015.

[25] HALTER-KOCH, F.-NARKIEWICZ, W.: Polynomial mappings defined by forms with a common factor, Sém. Théor. Nombres Bordeaux (2) 4 (1992), no. 2, 187-198.

[26] LANG, S.: Diophantine geometry, Interscience Tracts in Pure and Appl. Math. 11 (1962), pp. 170.

[27] _ Report on diophantine approximations, Bull. Soc. Math. France 93 (1965), 177-192.

[28] — : Division points on curves, Ann. Mat. Pura Appl. 70 (1965), no. 4, 229-234.

[29] LAURENT, M.: Équations diophantiennes exponentielles, Invent. Math. 78 (1984), 299-327.

[30] NARKIEWICZ, W.: Problem 415, Colloq. Math. 10 (1963), no. 1, p. 186.

[31] NARKIEWICZ, W.: On polynomial transformations. II, Acta Arith. 8 (1962/1963), $11-19$. 


\section{LES HUIT PREMIERS TRAVAUX DE PIERRE LIARDET}

[32] PILA, J.: o-minimality and the André-Oort conjecture for $\mathbb{C}^{n}$, Ann. of Math. (2) 173 (2011), no. 3, 1779-1840.

[33] POTTMEYER, L.: Heights and totally p-adic numbers, Acta Arith. 171 (2015), no. 3, $277-291$.

[34] RAUZY, G.: Transformations rationnelles pour lesquelles l'ensemble des nombres de Pisot-Vijayaraghavan est stable, C. R. Acad. Sci. Paris Sér. A-B 268 (1969), A305-A307.

[35] __ Ensembles de nombres algébriques et transformations rationnelles, in Colloque de Théorie des Nombres (Univ. Bordeaux, 1969), pp. 165-168. Bull. Soc. Math. France, Mém. Vol. 25, Soc. Math. France, Paris, 1971.

[36] SCANLON, T.: A proof of the André-Oort conjecture via mathematical logic [after Pila, Wilkie and Zannier], Séminaire Bourbaki Vol. 2010/2011. Exposés 1027-1042. Astérisque 348 (2012), Exp. No. 1037, ix, 299-315,

[37] TZERMIAS, P.: The Manin-Mumford conjecture: a brief survey, Bull. London Math. Soc. 32 (2000), no. 6, 641-652.

[38] ULlMO, E.: Structures spéciales et problème de Pink Zilber, Panorama et Synthèses (CIRM), to appear.

[39] WIDMER, M.: : On certain infinite extensions of the rationals with Northcott property, Monatsh. Math. 162 (2011), no. 3, 341-353.

[40] ZANNIER, U.: Some Problems of Unlikely Intersections in Arithmetic and Geometry, Annals of Mathematics Studies Vol. 181, Princeton University Press, Princeton, NJ, 2012.

Received June 1, 2016

Accepted October 10, 2016

\author{
Michel Waldschmidt \\ Sorbonne Universités \\ UPMC Univ Paris 06 \\ UMR 7586 IMJ-PRG \\ F-75005 Paris \\ FRANCE
}

E-mail: michel.waldschmidt@imj-prg.fr 